\title{
Orbital Properties of Regular Chain
}

\author{
Kaiguang Zhang, Haixia Du, Hongling Meng, Mingting Ba \\ Department of Mathematics, Zhengzhou Normal University, Zhengzhou, China \\ Email: zzgis@sina.com
}

Received 13 October 2014; revised 3 November 2014; accepted 16 November 2014

Copyright (C) 2014 by authors and Scientific Research Publishing Inc.

This work is licensed under the Creative Commons Attribution International License (CC BY).

http://creativecommons.org/licenses/by/4.0/

(c) (i) Open Access

\section{Abstract}

The strong Markov process had been obtained by Ray-Knight compacting; its orbit natures are discussed; the significance probability of kolmogorov forward and backward equations are explained.

\section{Keywords}

\section{Regular Chain, Regular State, Transient State, Predictable, Kolmogorov Forward and Backward Equation}

\section{Introduction}

General Markov chain only has locally strong Markov property, which is the main obstruction to solve the problem of Markov chain constructing [1] [2]. The papers construct a strong Markov chain corresponding to its transition function using Ray-Knight compact method [3] [4], which is named regular chain. The papers give an orbit construction of birth and death process [5] [6]. The papers solve the construction problem of two-sided birth and death process [3]-[11]. The papers prove that the appended points in the compacting and the points on the Martin entrance boundary are monogamy, under the condition of finite entrance boundary [12]-[14]. This paper makes a strong Markov process by Ray-Knight compacting, discusses its orbit nature and explains the significance probability of Kolmogorov forward and backward equations.

\section{The Orbit Natures of Regular Chain}

Assume $P(t)=\left(p_{i j}(t)\right)_{i, j \in E}$ is a honest transition function on $E=\{1,2, \cdots\}, Q=\left(q_{i j}\right)_{i, j \in E}$ is its density function, $R_{i j}(\lambda)$ is its resolvent, $\bar{E}$ is the Ray-Knight compacting of $E,\left(U^{\alpha}\right)_{\alpha>0}$ and $\left(P_{t}\right)_{t \geq 0}$ is the Ray resolvent and the semi-group correspondence, denote $D=\left\{x \mid x \in \bar{E}, P_{0}(x, \cdot)=\delta_{x}(\cdot)\right\}$ as non-ramification point set, $E_{R}=\left\{x \mid x \in \bar{E}, U^{1}(x, E)=1\right\}, E^{+}=E_{R} \cap D$, then $E$ is Borel algebras on $E^{+}, X=\left(\Omega, F, F_{t}, X_{t}, \theta_{t}, P^{x}\right)$ is the 
regular chain of correspondence to $P(t)$. Denote $T_{f l}=\inf \left\{s \mid s \geq 0, X_{s} \neq X_{0}\right\}$ and $T_{r e}=\inf \left\{s \mid s>0, X_{s}=X_{0}\right\}$ respectively as escape time and return time, by Blumenthal 0 - 1 law, for arbitrary $x \in E^{+}, P^{x}\left\{T_{f l}=0\right\}=0$ or 1 , $P^{x}\left\{T_{r e}=0\right\}$ or 1 , if $P^{x}\left\{T_{f l}=\infty\right\}=1, x$ is called absorption state, if $P^{x}\left\{T_{f l}>0\right\}=1, x$ is called sojourn state, if $P^{x}\left\{T_{r e}=0\right\}=1, x$ is called regular state, if $P^{x}\left\{T_{r e}=0\right\}=P^{x}\left\{T_{f l}=0\right\}=1, x$ is called temporary state.

Theorem 1 Let $i \in E$, then

(1) $i$ is a regular state,

(2) on $P^{i}$, the distribution of escape time $T_{f l}$ is the exponential distribution of $q_{i}$,

(3) on $P^{i}, X_{T_{f}}$ and $T$ is mutual independent.

(4) if $0<q_{i}<\infty$, for arbitrary $j \in E, j \neq i, P^{i}\left\{X_{T_{f l}}=j\right\}=\frac{q_{i j}}{q_{i}}$.

Proof (1) Assume $i$ is not a regular state, then $P^{i}\left\{T_{r e}>0\right\}=1$. for arbitrary $t>0$, it is easy to check $\left\{X_{t}=i\right\} \subseteq\left\{T_{r e} \leq t\right\}$, and when $t \rightarrow 0,\left\{T_{r e} \leq t\right\} \rightarrow\left\{T_{r e}=0\right\}$, thus

$$
1=\lim _{t \downarrow 0} p_{i i}(t)=\lim _{t \downarrow 0} P^{i}\left\{X_{t}=i\right\} \leq \lim _{t \downarrow 0} P^{i}\left\{T_{r e} \leq t\right\}=0,
$$

this is a contradictory proposition.

(2) The proof is same as Theorem 5 in [15].

(3) If $q_{i}=0$ or $\infty$, then $P^{i}\left\{T_{f l}=\infty\right\}=1$ or $P^{i}\left\{T_{f l}=0\right\}=1$, the conclusion is true, if $0<q_{i}<\infty$, for arbitrary Borel subset $A \subset E^{+}$and $t, s>0$,

$$
\begin{aligned}
& P^{i}\left\{T_{f l}>t+s, X_{T_{f l}} \in A\right\}=P^{i}\left\{T_{f l}>t, T_{f l} \circ \theta_{t}>s, X_{T_{f l}} \circ \theta_{t} \in A\right\} \\
& =E^{i}\left\{P^{X_{t}}\left\{T_{f l}>s, X_{T_{f l}} \in A\right\} ; T_{f l}>t\right\}=P^{i}\left\{T_{f l}>t\right\} P^{i}\left\{T_{f l}>s, X_{T_{f l}} \in A\right\} .
\end{aligned}
$$

Let $s \rightarrow 0$, we have $P^{i}\left\{T_{f l}>t, X_{T_{f l}} \in A\right\}=P^{i}\left\{T_{f l}>t\right\} P^{i}\left\{X_{T_{f l}} \in A\right\}$.

then, on $P^{i}, P^{i}, X_{T_{f l}}$ and $T$ is mutual independent.

(4) If $0<q_{i}<\infty$, for arbitrary $j \neq i, \lambda>0$, According to the strong Markov properties of $X$ and (3), we can obtain that

$$
\begin{aligned}
R_{i j}(\lambda) & =\int_{0}^{\infty} \mathrm{e}^{-\lambda} p_{i j}(t) \mathrm{d} t=E^{i}\left[\int_{0}^{\infty} \mathrm{e}^{-\lambda t} I_{\{j\}}\left(X_{t}\right) \mathrm{d} t\right]=E^{i}\left[\int_{T_{f l}}^{\infty} \mathrm{e}^{-\lambda t} I_{\{j\}}\left(X_{t}\right) \mathrm{d} t\right] \\
& =E^{i}\left[\mathrm{e}^{-\lambda T_{f l}}\left[\int_{0}^{\infty} \mathrm{e}^{-\lambda t} I_{\{j\}}\left(X_{t}\right) \mathrm{d} t\right] \circ \theta_{T_{f l}}\right]=E^{i}\left[\mathrm{e}^{-\lambda T_{f l}} E^{X_{T_{f f}}}\left[\int_{0}^{\infty} \mathrm{e}^{-\lambda t} I_{\{j\}}\left(X_{t}\right) \mathrm{d} t\right]\right] \\
& =E^{i}\left[\mathrm{e}^{-\lambda T_{f l}} \int_{0}^{\infty} \mathrm{e}^{-\lambda t} \mathrm{P}^{X_{T_{f f}}}\left[X_{t}=j\right] \mathrm{d} t\right]=E^{i}\left[\mathrm{e}^{-\lambda T_{f f}} \int_{0}^{\infty} \mathrm{e}^{-\lambda t} P_{t}\left(X_{T_{f l}},\{j\}\right) \mathrm{d} t\right] \\
& =E^{i}\left[\mathrm{e}^{-\lambda T_{f f}} U^{\lambda}\left(X_{T_{f l}},\{j\}\right)\right]=E^{i}\left[\mathrm{e}^{-\lambda T_{f l}}\right] E^{i}\left[U^{\lambda}\left(X_{T_{f l}},\{j\}\right)\right]
\end{aligned}
$$

Give arbitrary $x \in E^{+}, x \neq j$, and continuous function $f(\cdot)$ on $\bar{E}$ with $f(x)=0, f(j)=1$,

$$
0=f(x)=\lim _{\lambda \rightarrow \infty} \lambda U^{\lambda} f(x) \geq \lim _{\lambda \rightarrow \infty} \lambda U^{\lambda}(x,\{j\}),
$$

but $\lim _{\lambda \rightarrow \infty} \lambda U^{\lambda}(j,\{j\})=\lim _{\lambda \rightarrow \infty} \lambda R_{j j}(\lambda)=1$, in addition,

$$
\begin{aligned}
q_{i j} & =\lim _{\lambda \rightarrow \infty} \lambda^{2} R_{i j}(\lambda)=\lim _{\lambda \rightarrow \infty} \lambda E^{i}\left[\mathrm{e}^{-\lambda T_{f}}\right] \lim _{\lambda \rightarrow \infty} \lambda E^{i}\left[U^{\lambda}\left(X_{T_{f}},\{j\}\right)\right] \\
& =\lim _{\lambda \rightarrow \infty} \frac{\lambda q_{i}}{\lambda+q_{i}} E^{i}\left[\lim _{\lambda \rightarrow \infty} U^{\lambda}\left(X_{T_{f}},\{j\}\right)\right]=q_{i} P^{i}\left[X_{T_{f}}=j\right],
\end{aligned}
$$

thus, $P^{i}\left\{X_{T_{f l}}=j\right\}=\frac{q_{i j}}{q_{i}}$.

Remark 1 (3), (4) in the Theorem 1 are equivalence with the Theorem 6 in [15], but it require $q_{j}<\infty$, do 
not incloude $q_{j}=\infty$.

Remark 2 According to (2) in Theorem 1, $i \in E$ is a temporary state, if and only if $i$ is a sojourn state of the regular chain $X=\left(\Omega, F, F_{t}, X_{t}, \theta_{t}, P^{x}\right)$.

Definition 1 Let $S_{i}(\omega)=\left\{s \mid X_{s}(\omega)=i\right\}$ is the constant set of $i$, the interval in $S_{i}(\omega)$ is called i-interval of $X$.

Theorem 2 If $q_{i}<\infty$, then for arbitrary $x \in E^{+}$, we can get a stopping time squence $a_{1}, b_{1}, a_{2}, b_{2}, \cdots$, with $a_{k} \leq b_{k}$, when $\left\{a_{k}<\infty\right\}$, we have $a_{k}<b_{k}$, when $\left\{b_{k}<\infty\right\}$, we have $b_{k}<a_{k+1}$. And for arbitrary $k$,

$$
S_{i}(\omega)=\bigcup_{k}\left[a_{k}(\omega), b_{k}(\omega)\right), P^{x} \text { a.s.. }
$$

For arbitrary $s<t$, denote $\xi_{i}(s, t)$ as the number of $\left[a_{k}, b_{k}\right)$ belong to $[s, t]$, we have

$$
E^{x}\left\{\xi_{i}(s, t)\right\} \leq q_{i}(t-s) .
$$

\section{Proof Let}

$$
\begin{aligned}
& a_{1}=\inf \left\{u \mid u \geq 0, X_{u}=i\right\}, b_{1}=\inf \left\{u \mid u \geq a_{1}, X_{u} \neq i\right\}, \\
& a_{k+1}=\inf \left\{u \mid u \geq b_{k}, X_{u}=i\right\}, \quad b_{k+1}=\inf \left\{u \mid u \geq a_{k}, X_{u} \neq i\right\}, \quad k=1,2, \cdots,
\end{aligned}
$$

where $a_{k}, b_{k}, k=1,2, \cdots$ are the stoping time of $\left\{F_{t}\right\}$. for arbitrary $k$, if $a_{k}<\infty$, since $X$ is right continuity, $X_{a_{k}}=i$, and

$$
\begin{aligned}
P^{x}\left[a_{k}<\infty, b_{k}>a_{k}\right] & =P^{x}\left[a_{k}<\infty, T_{f l} \circ \theta a_{k}>0\right] \\
& =E^{x}\left[P^{x}\left[T_{f l} \circ \theta a_{k}>0 \mid F a_{k}\right], a_{k}<\infty\right] \\
& =E^{x}\left[P^{i}\left[T_{f l}>0\right], a_{k}<\infty\right]=P^{x}\left[a_{k}<\infty\right]
\end{aligned}
$$

then we have almost sure $a_{k}<b_{k}$ on $\left\{a_{k}<\infty\right\}$.

Since $X$ is strong Markov chain, and for arbitrary $k$,

$$
\begin{aligned}
& P^{x}\left[b_{k}<\infty, \exists \varepsilon>0, X=i, \text { in }\left[b_{k}, b_{k}+\varepsilon\right)\right] \\
& =P^{x}\left[b_{k}<\infty, X_{b_{k}}=i, T_{f l} \circ \theta b_{k}>0\right] \\
& =E^{x}\left[P^{x}\left[T_{f l} \circ \theta b_{k}>0 \mid F b_{k}\right] ; b_{k}<\infty, X_{b_{k}}=i\right] \\
& =E^{x}\left[P^{i}\left[T_{f l}>0\right] ; b_{k}<\infty, X_{b_{k}}=i\right] \\
& =P^{x}\left[X_{b_{k}}=i, b_{k}<\infty\right]
\end{aligned}
$$

then we have almost sure $X_{b_{k}} \neq i$ on $\left\{b_{k}<\infty\right\}$.

For arbitrary $0<s<t$, obviously $\xi_{i}(s, t)=\xi_{i}(0, t-s) \circ \theta_{s}$, by Theorem 3.1 in [15]

$$
E^{x}\left\{\xi_{i}(s, t)\right\} \leq E^{x}\left\{\xi_{i}(0, t-s) \circ \theta_{s}\right\}=\sum_{k \in E} P^{x}\left\{X_{s}=k\right\} E^{k}\left\{\xi_{i}(0, t-s)\right\} \leq q_{i} \cdot(t-s) .
$$

According to Fatou lemma, for arbitrary $t>0, E^{x}\left\{\xi_{i}(0, t)\right\} \leq q_{i} \cdot t$, then almost sure there are only finite $\left[a_{k}, b_{k}\right), k=1,2, \cdots$ in a finite interval, such that $\lim _{k \rightarrow \infty} a_{k}=\infty$, this means $S_{i}(\omega)=\bigcup_{k}\left[a_{k}(\omega), b_{k}(\omega)\right)$.

Theorem 3 If $q_{i}=\infty$, then

(1) Almost sure, $S_{i}(\omega)$ do not contain any interval,

(2) Almost sure, $S_{i}(\omega)$ is a dense set in itself.

Proof (1) Obviously, $S_{i}(\omega)$ is a optional set, denote $A_{t}(\omega)=\sup \left\{s \mid s<t, s \notin S_{i}(\omega)\right\}, t \geq 0, \omega \in \Omega$. (where we assume $\sup \varnothing=0)$, then $\left\{A_{t}\right\}$ is a monotone increasing left continuous process, and adapt in $\left\{F_{t}\right\}$, denote $B_{t}=\lim _{s \downarrow t} A_{s}$, thus $\left\{B_{t}\right\}$ is a optional right continuous process. Let 


$$
U=\left\{(\omega, t) \mid \exists \varepsilon>0, \ni(t-\varepsilon, t+\varepsilon) \subseteq S_{i}(\omega)\right\}, \omega \in \Omega .,
$$

It is easy to check that $U=\left\{(\omega, t) \mid B_{t}(\omega)<t\right\}$, thus $U$ is a optional set adapt in $\left\{F_{t}\right\}$.

Assume $D_{U}$ is debut time, If $P^{x}\left\{D_{U}<\infty\right\}>0$, by Section Theorem, exists a stopping time $T$ in $\left\{F_{t}\right\}$, such that $P^{x}\{T<\infty\}>0$, and $(\omega, T(\omega)) \in U$ on $\{T<\infty\}$, by (2) in the theorem 1,

$$
\begin{aligned}
P^{x}[T<\infty] & =P^{x}\left[T<\infty, X_{T}=i, \exists \varepsilon>0, \text { э } X \equiv i \text { on }(T-\varepsilon, T+\varepsilon)\right] \\
& \leq P^{x}\left[T<\infty, X_{T}=i, T_{f l} \circ \theta_{T}>0\right]=E^{x}\left[P^{i}\left[T_{f l}>0\right] ; T<\infty\right]=0
\end{aligned}
$$

this is a contradictory proposition, thus $P^{x}\left\{D_{U}<\infty\right\}=0$ and almost sure $S_{i}(\omega)$ do not contain any interval.

(2) The proof is similar to (1).

\section{The Significance Probability of Kolmogorov Equations}

Theorem 4 For arbitrary $i \in E, j \in E$ and $t \geq 0$

$$
p_{i j}^{\prime}(t)=-q_{i} p_{i j}(t)+\sum_{k \neq i} q_{i k} p_{k j}(t)
$$

if and only if $P^{i}\left\{X_{T_{f l}} \in E\right\}=1$.

Proof For arbitrary $\lambda>0$,

$$
\begin{gathered}
\int_{0}^{\infty} \mathrm{e}^{-\lambda t}\left[-q_{i} p_{i j}(t)+\sum_{k \neq i} q_{i k} p_{k j}(t)\right]=-q_{i} R_{i j}(\lambda)+\sum_{k \neq i} q_{i k} R_{k j}(\lambda) \\
\int_{0}^{\infty} \mathrm{e}^{-\lambda t} p_{i j}^{\prime}(t) \mathrm{d} t=\int_{0}^{\infty} \mathrm{e}^{-\lambda t} \mathrm{~d} p_{i j}^{\prime}(t)=-\delta_{i j}+\lambda \int_{0}^{\infty} p_{i j}(t) \mathrm{d} t=-\delta_{i j}+\lambda R_{i j}(\lambda)
\end{gathered}
$$

then (1) and the following equation is equivalence.

$$
\left(\lambda+q_{i}\right) R_{i j}(\lambda)-\sum_{k \neq i} q_{i k} R_{k j}(\lambda)=\delta_{i j}, \lambda>0, j \in E
$$

According to Theorem 1, we have

$$
\begin{aligned}
& R_{i j}(\lambda)=E^{i}\left[\int_{0}^{\infty} \mathrm{e}^{-\lambda t} I_{\{j\}}\left(X_{t}\right) \mathrm{d} t\right] \\
& =E^{i}\left[\int_{0}^{T_{f l}} \mathrm{e}^{-\lambda t} \delta_{i j} \mathrm{~d} t\right]+E^{i}\left[\int_{T_{f l}}^{\infty} \mathrm{e}^{-\lambda t} I_{\{j\}}\left(X_{t}\right) \mathrm{d} t\right] \\
& =\frac{1}{\lambda} E^{i}\left[1-\mathrm{e}^{-\lambda T_{f l}}\right] \cdot \delta_{i j}+E^{i}\left[\mathrm{e}^{-\lambda T_{f l}} \cdot\left[\int_{0}^{\infty} \mathrm{e}^{-\lambda t} I_{\{j\}}\left(X_{t}\right) \mathrm{d} t\right] \cdot \theta_{T_{f l}}\right] \\
& =\frac{1}{\lambda} E^{i}\left[1-\mathrm{e}^{-\lambda T_{f l}}\right] \cdot \delta_{i j}+E^{i}\left[\mathrm{e}^{-\lambda t T_{f}} U^{\lambda}\left(X_{T_{f f}},\{j\}\right)\right] \\
& \geq \frac{1}{\lambda} E^{i}\left[1-\mathrm{e}^{-\lambda T_{f l}}\right] \cdot \delta_{i j}+E^{i}\left[\mathrm{e}^{-\lambda t T_{f l}}\right] E^{i}\left[U^{\lambda}\left(X_{T_{f f}},\{j\}\right) ; X_{T_{f l}} \in E\right] \\
& =\frac{\delta_{i j}}{\lambda+q_{i}}+\frac{q_{i j}}{\lambda+q_{i}} \sum_{k \neq j} \frac{q_{i k}}{q_{i}} R_{k j}(\lambda)=\frac{\delta_{i j}+\sum_{k \neq j} q_{i k} R_{k j}}{\lambda+q_{i}}
\end{aligned}
$$

and the necessary and sufficient condition of equality is $P^{i}\left\{X_{T_{f l}} \in E\right\}=1$.

For arbitrary $i \in E, q_{i}<\infty$, let $\left[a_{k}^{(i)}, b_{k}^{(i)}\right)$ is the first k i-interval of $S_{i}(\omega)$,

$$
S_{\infty}^{-}(\omega)=\{t \mid t>0 \text {, for arbitrary } \varepsilon>0,(t-\varepsilon, t) \text { have infinite jumps }\}
$$

Corollary 1 The following conditions are equivalence [16] [17]. 
(1) The backward equation of Kolmogorov is true,

(2) For arbitrary $i \in E, P^{i}\left\{X_{T_{f}} \in E\right\}=1$,

(3) Density matrix $Q$ is conservative,

(4) Almost sure, for all $i \in E$ and $\left[a_{k}^{(i)}, b_{k}^{(i)}\right)$, we have $X_{b_{k}^{(i)}} \in E$.

Theorem 5 For arbitrary $i, j \in E$

$$
p_{i j}^{\prime}(t)=-p_{i j}(t) q_{j}+\sum_{k \neq j} p_{i k}(t) q_{k j}, \forall t \geq 0
$$

if and only if for all $j$-interval $\left[a_{l}^{(j)}, b_{l}^{(j)}\right)$ almost sure $a_{k}^{(i)} \notin S_{\infty}^{-}$.

Proof (1) Asumme $t_{1}<t_{2}, k \in E, k \neq j, n \in \mathrm{N}$,

$$
\begin{gathered}
U=\left\{\exists l, t_{1}<a_{l}^{(j)}<t_{2}<b_{l}^{(j)}, a_{l}^{(j)} \notin S_{\infty}^{-}, X_{a_{l}^{(j)}-}=k\right\}, s_{m}^{n}=t_{1}+\frac{m}{n}\left(t_{2}-t_{1}\right), \\
U_{m}^{n}=\left\{X_{s_{m}^{n}}=k, X . \equiv j \text { on }\left[s_{m}^{n}+\frac{t_{2}-t_{1}}{n}, t_{2}\right]\right\}, m=0,1,2, \cdots, n-1, U^{n}=\bigcup_{m=0}^{n-1} U_{m}^{n} .
\end{gathered}
$$

Obviously $U_{0}^{n}, U_{1}^{n}, \cdots, U_{n}^{n-1}$ are not intersection. It is easy to check if there are infinite $n$ to make $\omega \in U^{n}$, then $\omega \in U$, and if $\omega \in U$, then existing $N$, when $n>N$, we have $\omega \in U^{n}$, thus that $\lim _{n \rightarrow \infty} U^{n}=U$, and

$$
P^{i}\{U\}=\lim _{n \rightarrow \infty} P^{i}\left\{U^{n}\right\}=\lim _{n \rightarrow \infty} \sum_{m=0}^{n-1} P^{i}\left\{U_{m}^{n}\right\}=\lim _{n \rightarrow \infty} \sum_{m=0}^{n-1} p_{i k}\left(s_{m}^{n}\right) p_{k j}\left(\frac{t_{2}-t_{1}}{n}\right) \mathrm{e}^{-q_{j}\left(t_{2}-s_{m}^{n}-\frac{t_{2}-t_{1}}{n}\right)}=\int_{t_{1}}^{t_{2}} p_{i k}(s) q_{k j} \mathrm{e}^{-q_{i}(t-s)} \mathrm{d} s .
$$

(2) For arbitrary $t_{1}<t_{2}$, by (1),

$$
\begin{aligned}
p_{i j}\left(t_{2}\right) & =P^{i}\left[\exists l, a_{l}^{(j)}<t_{2}<b_{l}^{(j)}\right] \\
& =P^{i}\left[\exists l, a_{l}^{(j)} \leq t_{1}<t_{2}<b_{l}^{(j)}\right]+P^{i}\left[\exists l, t_{1}<a_{l}^{(j)}<t_{2}<b_{l}^{(j)}\right] \\
& \geq P_{i j}\left(t_{1}\right) \mathrm{e}^{-q_{i}\left(t_{2}-t_{1}\right)}+P^{i}\left[\exists l, t_{1}<a_{l}^{(j)}<t_{2}<b_{l}^{(j)}, a_{l}^{(j)} \notin S_{\infty}^{-}\right] \\
& =P_{i j}\left(t_{1}\right) \mathrm{e}^{-q_{i}\left(t_{2}-t_{1}\right)}+\sum_{k \neq j} P^{i}\left[\exists l, t_{1}<a_{l}^{(j)}<t_{2}<b_{l}^{(j)}, a_{l}^{(j)} \notin S_{\infty}^{-}, X_{\left.a_{l}^{(j)}\right)_{-}}=k\right] \\
& =P_{i j}\left(t_{1}\right) \mathrm{e}^{-q_{i}\left(t_{2}-t_{1}\right)}+\sum_{k \neq j} \int_{t_{1}}^{t_{2}} p_{i k}(s) q_{k j} \mathrm{e}^{-q_{i}^{(t-s)}} \mathrm{ds}
\end{aligned}
$$

and the necessary and sufficient condition of equality is $P^{i}\left\{\forall l, a_{l}^{(j)} \notin S_{\infty}^{-}\right\}=1$.

Thus we get the equation

$$
\frac{p_{i j}\left(t_{2}\right)-p_{i j}\left(t_{1}\right) \mathrm{e}^{-q_{i}\left(t_{2}-t_{1}\right)}}{t_{2}-t_{1}}=\frac{\sum_{k \neq j} \int_{t_{1}}^{t_{2}} p_{i k}(s) q_{k j} \mathrm{e}^{-q_{i}(t-s)} \mathrm{d} s}{t_{2}-t_{1}},
$$

let $t_{2}$ go to $t_{1}$ in Equation (5), we can obtain Equation (3).

Corollary 2 The Kolmogorov forward equations are true if and only if for all $i \in E$ and $i$-interval $\left[a_{k}^{(i)}, b_{k}^{(i)}\right)$, almost sure $a_{k}^{(i)} \notin S_{\infty}^{-}$.

Remark 3 Equation(3) is equivalent to

$$
R_{i j}(\lambda) \cdot\left(\lambda+q_{j}\right)-\sum_{k \neq j} R_{i k}(\lambda) q_{k j}=\delta_{i j}, \forall \lambda>0 .
$$

Remark 4 If $P(t)$ contains some transient state, then Equation (1) is true if and only if

$$
P^{i}\left[\forall l, \exists k \in E,\left\{s_{n}\right\}_{n=1}^{\infty}, \ni s_{n} \uparrow a_{l}^{(j)}, X_{s_{n}}=k\right]=1
$$


Remark 5 Under the condition of $P^{i}\left\{\forall l, X_{a_{l}^{(j)}-} \in E\right\}=1$, Equation (1) is not probably true. for the example in Remark 1, the Ray-Knight compaction of $E$ under the resolvent $R_{i j}(\lambda)$ is $E$, thus, the corresponding regular chain meets the equation $P^{i}\left[\forall l, X_{a_{l}^{i()-}} \in E\right]=1$, but according to Corollary 2, Doob process does not satisfy Kolmogorov forward equation, then $R_{i j}(\lambda)$ also does not satisfy forward equation.

If $P(t)=\left(p_{i j}(t)\right)_{i, j \in E}, t \geq 0$ is an non-honest transition function with total stability, then we can construct a honest transition function $\bar{P}(t)=\left(\bar{p}_{i j}(t)\right)_{i, j \in E_{\Delta}}, t \geq 0$ on $E_{\Delta}=E \cup\{\Delta\}$, such that

$$
p_{\Delta \Delta}(t)=1, p_{\Delta i}(t)=0, p_{i \Delta}=1-\sum_{k \in E} p_{i k}(t), \forall i \in E,
$$

where the density matrix of $\bar{P}(t)$ is $\bar{Q}=\left(\bar{q}_{i j}(t)\right)_{i, j \in E_{\Delta}}$, such that

$$
\overline{q_{\Delta}}=0, \overline{q_{\Delta j}}=0, \overline{q_{i j}}=q_{i j}, \forall i, j \in E
$$

the resolvent of $\bar{P}(t)$ is $\bar{R}_{i j}(\lambda)$, then

$$
\overline{R_{\Delta \Delta}}(\lambda)=\frac{1}{\lambda}, \overline{R_{\Delta j}}(\lambda)=0, \overline{R_{i j}}(\lambda)=R_{i j}(\lambda), \forall i, j \in E
$$

Assume $X=\left(\Omega, F, F_{t}, X_{t}, \theta_{t}, P^{x}\right)$ is a regular chain corresponding to $\bar{P}(t)$. For $\bar{q}_{\Delta}(t)=0$, by Theorem 1 , $\Delta$ is a absorption state, this is $p^{\Delta}\left\{X_{t}=\Delta, \forall t \geq 0\right\}=1$.

Set $\xi=\inf \left\{s \mid X_{s}=\Delta\right\}, X^{\xi}=\left\{X_{t} \mid t<\xi\right\}$, obviously $X^{\xi}$ is a killing Markov process, for arbitrary $i, j \in E$, $\lambda>0$, and $R_{i j}(\lambda)=\overline{R_{i j}}(\lambda)=\int_{0}^{\infty} \mathrm{e}^{-\lambda t} P^{i}\left\{X_{t}=j\right\} \mathrm{d} t=\int_{0}^{\infty} \mathrm{e}^{-\lambda t} P^{i}\left\{X_{t}=j, t<\xi\right\} \mathrm{d} t$, we known the transition function of $X^{\zeta}$ is $P(t)$.

For arbitrary $i, j \in E$, since $\overline{p_{\Delta j}}=0, \overline{p_{i j}}(t)=p_{i j}(t)$, then for arbitrary $t \geq 0$ the following equations are Equivalence.

$$
\begin{aligned}
& \overline{p_{i j}^{\prime}}(t)=-\overline{p_{i j}}(t) \overline{q_{j}}+\sum_{k \in E_{\Delta}, k \neq j} \overline{p_{i k}}(t) \overline{q_{k j}}, \\
& p_{i j}^{\prime}(t)=-p_{i j}(t) q_{j}+\sum_{k \in E, k \neq j} p_{i k}(t) q_{k j} .
\end{aligned}
$$

It is easy to get:

Proposition 1 Assume $P(t)=\left(p_{i j}(t)\right)_{i, j \in E}$ is an non-honest transition function with total stability, $X^{\xi}$ is corresponding Markov process with killing, then $P(t)$ satisfy Kolmogorov backward equation if and only if almost sure for all $i \in E$ and $i$-interval, $X_{b_{k}^{(i)}} \in E_{\Delta}$.

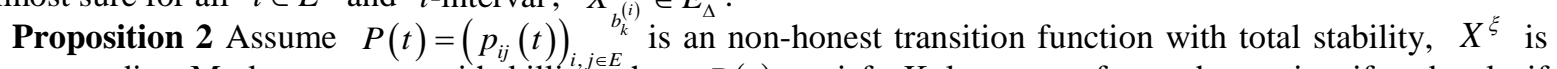
corresponding Markov process with killing, then $P(t)$ satisfy Kolmogorov forward equation if and only if almost sure for all $i \in E$ and $i$-interval, $a_{k}^{(i)} \notin S_{\infty}^{-}$.

\section{References}

[1] Rockner, M. and Wang, F.Y. (2004) Weak Poincare Inequalities and L ${ }^{2}$-Convergence Rates of Markov Semi-Groups. Journal of Functional Analysis, 185, 564-603. http://dx.doi.org/10.1006/jfan.2001.3776

[2] Xie, F.Y., Wu, B. and Hu, Y.M. (2013) A Generalized Markov Chain Model Based on Generalized Interval Probability. Science China Technological Sciences, 56, 2132-2136. http://dx.doi.org/10.1007/s11431-013-5285-3

[3] Dong, J.C. (2006) Ray-Knight Compactification of Markov Chain. Ph.D., Zhengzhou University, Zengzhou.

[4] Xiao, Y.M. (2014) Criterion of Semi-Markov Dependent Risk Model. Acta Mathematica Sinica (English Series), 30, 1273-1280. http://dx.doi.org/10.1007/s10114-014-3249-6

[5] Barthe, F., Cattiaux, P. and Roberto, C. (2007) Isoperimetry between Exponential and Gaussian. Electronic Journal of Probability, 12, 1212-1237. http://dx.doi.org/10.1214/EJP.v12-441

[6] Barthe, F., Cattiaux, P. and Roberto, C. (2006) Interpolated Inequalities between Exponential and Gaussian, Orlicz 
Hyper Contractivity and Isoperimetry. Revista Matemática Iberoamericana, 22, 993-1066. http://dx.doi.org/10.4171/RMI/482

[7] Chen, L. (2012) Paths of Bilateral Birtth-Death Processes and Construction Theory(I). Journal of Henan University (Nature Science), 42, 337-342.

[8] Zhang, L.C. and Guo, M.Z. (2014) The Characterization of a Class of Quantum Markov Semi-Groups and the Associated Operator-Valued Dirichlet Forms Based on Hilbert C*-Module 12(A). Science China Mathematics, 57, 377-387. http://dx.doi.org/10.1007/s11425-013-4678-x

[9] Chen, L. (2013) Paths of Bilateral Birth-Death Processes and Construction Theory(II). Journal of Henan University (Nature Sciencd), 43, 5-10.

[10] Zou, B., Xu, Z.B. and Xu, J. (2014) Generalization Bounds of ERM Algorithm with Markov Chain Samples. Acta Mathematicae Applicatae Sinica (English Series), 30, 223-238. http://dx.doi.org/10.1007/s10255-011-0096-4

[11] Shimomura, H. (1994) Poisson Measures on Configuration Space and Unitary Representation of the Group of Diffeomorphisms. Journal of Mathematics of Kyoto University, 34, 599-614.

[12] Xu, C.W. and Yan, G.J. (2011) Martin Entrance Boundary and Ray-Knight Compactification of Minimal Q-Processes. Chinese Journal of Applied Probability and Statistics, 27, 633-641.

[13] Yang, W.J. (2014) Some Researches of Strong Limit Theorems for Markov Chains Indexed by Trees. Advances in Mathematics (China), 32, 206-218.

[14] Wen, S.F., Xu, M. and Wang, F.L. (2014) A New Method to Estimate Markov State Transition Probability Matrix. Mathematics in Practice and Theory, 44, 164-168.

[15] Wang, Z.K. (2005) Birth-Death Processes and Markov Chain. Science Press, Beijing.

[16] Xiang, X.Y. (2013) The Q-Matrix of Ring Markov Chain. ACTA Mathematica Sinica (Chinese Series), 56, 735-750.

[17] Luckock, H. (2003) A Steady-State Model of the Continuous Double Auction. Quantitative Finance, 3, 385-404. http://dx.doi.org/10.1088/1469-7688/3/5/305 
Scientific Research Publishing (SCIRP) is one of the largest Open Access journal publishers. It is currently publishing more than 200 open access, online, peer-reviewed journals covering a wide range of academic disciplines. SCIRP serves the worldwide academic communities and contributes to the progress and application of science with its publication.

Other selected journals from SCIRP are listed as below. Submit your manuscript to us via either submit@scirp.org or Online Submission Portal.
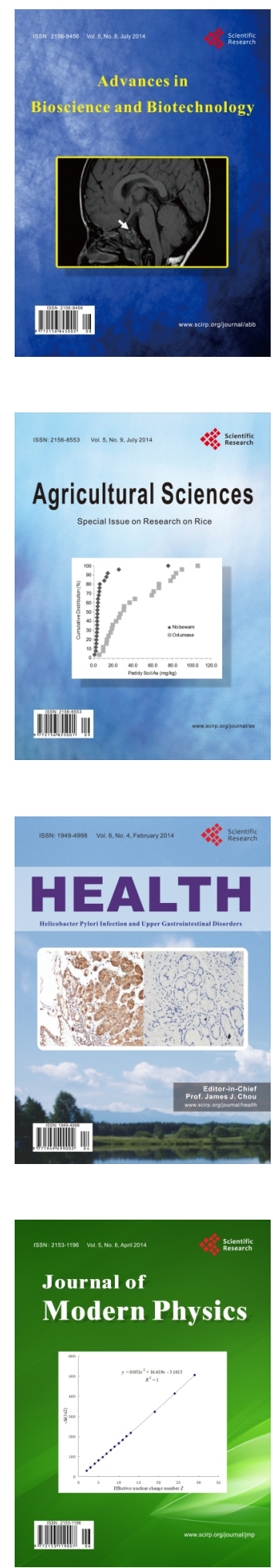
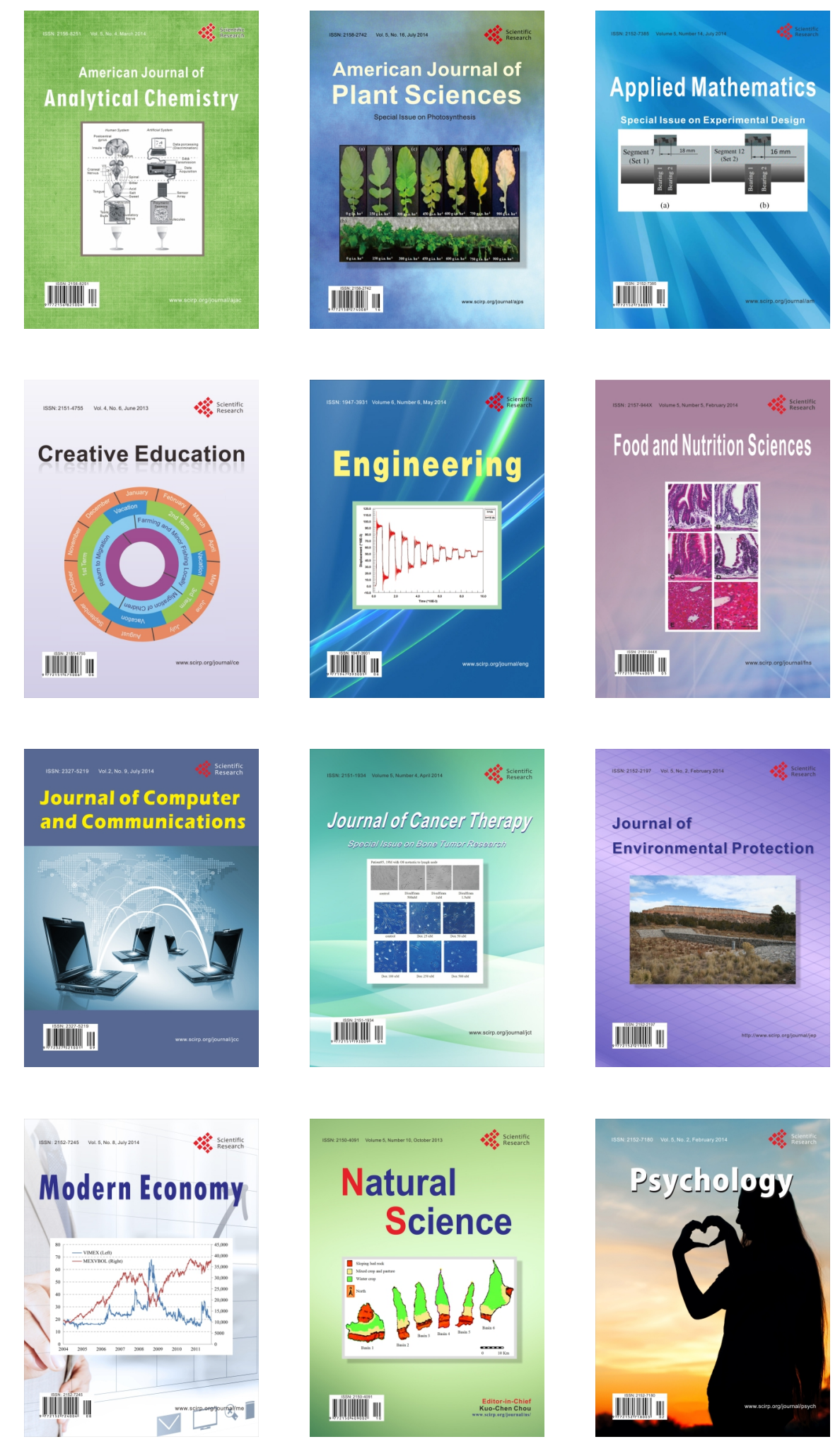